\title{
BMJ Open Measurement of coverage, compliance and determinants of uptake in a publicly funded rotavirus vaccination programme: a retrospective cohort study
}

\author{
Ellen Rafferty, ${ }^{1}$ Xiaoyan Guo, ${ }^{2}$ Bruce McDonald, ${ }^{2}$ Lawrence W Svenson (D) , 2,3 \\ Shannon Elizabeth MacDonald ${ }^{1,4}$
}

To cite: Rafferty $\mathrm{E}$,

Guo X, McDonald B, et al.

Measurement of coverage, compliance and determinants of uptake in a publicly funded rotavirus vaccination programme: a retrospective cohort study. BMJ Open 2019;9:e031718. doi:10.1136/ bmjopen-2019-031718

- Prepublication history for this paper is available online. To view these files, please visit the journal online (http://dx.doi org/10.1136/bmjopen-2019031718).

Received 16 May 2019 Revised 28 September 2019 Accepted 03 October 2019

Check for updates

(C) Author(s) (or their employer(s)) 2019. Re-use permitted under CC BY-NC. No commercial re-use. See rights and permissions. Published by BMJ.

${ }^{1}$ Nursing, University of Alberta, Edmonton, Alberta, Canada ${ }^{2}$ Analytics and Performance Reporting Branch, Alberta Ministry of Health, Edmonton, Alberta, Canada

${ }^{3}$ Faculty of Medicine and Dentistry, University of Alberta, Edmonton, Alberta, Canada

${ }^{4}$ School of Public Health,

University of Alberta, Edmonton, Alberta, Canada

Correspondence to Dr Shannon Elizabeth

MacDonald;

smacdon@ualberta.ca

\section{ABSTRACT}

Objectives In June 2015, Alberta, Canada instituted a universal publicly funded rotavirus vaccination programme (Rotarix, RV1), with vaccine doses scheduled for 2 and 4 months of age. Vaccination was restricted so that infants were only allowed to receive first dose between 6 and 20 weeks of age, and second dose before eight calendar months of age. We assessed the coverage and schedule non-compliance of rotavirus vaccination for babies born between June 2015 and August 2016, that is, since the inception of the publicly funded rotavirus vaccination programme, and determined factors associated with rotavirus vaccine uptake.

Design Retrospective cohort study using linked administrative health data.

Setting Alberta, Canada.

Participants Cohort of 66689 children.

Primary and secondary outcome measures (1) First

and second dose rotavirus vaccination coverage, (2) percent of children non-compliant with recommended vaccine schedule and (3) adjusted ORs for factors associated with vaccination status.

Results For the 66689 children included in the study, coverage levels for one-dose and two-dose rotavirus vaccination were $87 \%$ and $83 \%$, respectively. In comparison, two-dose diphtheria-tetanus-pertussis-polioHaemophilus influenzae type $b$ vaccine coverage was $92 \%$, despite having the same dosing schedule. Schedule non-compliance during the publicly funded programme was very low. We observed socioeconomic disparities in the uptake of the vaccine, with income, location of residence and number of children in the household all contributing to the odds of a child being vaccinated with rotavirus.

Conclusions Compliance to the recommended rotavirus schedule was very high, suggesting that even with the restrictive rotavirus vaccine schedule, the vaccine can be delivered on-time. However, rotavirus vaccine coverage remained lower than DTaP, a similarly scheduled childhood vaccination. We also observed socioeconomic disparities in vaccine uptake. These findings raise concerns about rotavirus protection in the groups at highest risk for gastrointestinal illness, including low-income and rural populations.
Strengths and limitations of this study

- We used a population-based immunisation repository of $>66000$ children in a context of a single vaccine provider (public health), which precluded record scattering

- We were able to deterministically link multiple administrative data sources using unique lifetime identifiers to assess characteristics of non-immunised children.

- We were able to assess compliance to the restricted rotavirus vaccine schedule as we had exact date of vaccine administration.

- We were unable to include First Nations (Indigenous) children in the study, because these records are not in the repository.

- We only had a limited study period (14 months) reducing our ability to investigate changes over time.

\section{INTRODUCTION}

Rotavirus was the leading cause of severe gastroenteritis in children under 5 in the world in 2016. ${ }^{1}$ Prior to vaccination, nearly every child was infected with rotavirus by the time they turned 5 years old, with the majority of infections, and the most severe cases, occurring in infancy. ${ }^{2}$ In fact, over two million children were hospitalised for rotavirus in the world each year, and $\sim 5 \%$ of all childhood deaths were attributable to rotavirus gastroenteritis. ${ }^{2}$ In developed countries like Canada, rotavirus does not typically result in death, likely because of access to medical care, in particular hydration therapy, good nutrition and a lower risk of concomitant infections. ${ }^{3}$ However, it does lead to severe illness and hospitalisations in young infants; resulting in a significant burden of disease on the children and their families, as well as a high economic burden. ${ }^{4-6}$

There are two rotavirus vaccines available internationally. Rotarix (RV1, manufactured 
by GlaxoSmithKline) is a monovalent vaccine requiring two doses. RotaTeq (RV5, manufactured by Merck) is a pentavalent vaccine requiring three doses. ${ }^{2}$ Estimates of the effectiveness of RV1 and RV5 suggest that if vaccine coverage is high, the vaccine is effective at preventing moderate and high severity rotavirus cases in highincome countries. ${ }^{3} 78$ For instance, multiple studies found that three doses of RV5 were between $83 \%$ and 92\% effective at preventing emergency department visits and hospitalisation in infants, and two doses of RV1 had an overall vaccine effectiveness between $92 \%$ and $98 \%$ in high-income countries. ${ }^{3}$ Furthermore, studies show up to 3 years of sustained protection postvaccination. ${ }^{7}$ There are concerns about the risk of intussusception in infants following rotavirus vaccination. The risk of this adverse event is very low, ${ }^{910}$ but there is concern the risk could increase if the vaccine is administered outside of the recommended schedule (eg, $>8$ months). ${ }^{3}$

An increasing number of countries are including rotavirus vaccine in their publicly funded vaccination programmes. ${ }^{11}$ In Canada, almost all provinces and territories have a universal rotavirus programme. The province of Alberta started universal rotavirus vaccination in June 2015 with RV1 being publicly funded and provided through public health clinics. RV5 was not publicly funded in Alberta during this period, although it was privately available. Prior to universal vaccination in Alberta, both the RV1 and RV5 vaccines were available privately, and individuals either purchased the vaccine out-of-pocket or received it through a private health insurance programme.

Measuring rotavirus vaccine coverage and the factors that contribute to coverage is important for vaccine programme planning and evaluation, to ensure high levels of coverage are reached and sustained. Furthermore, determining compliance levels can help clinicians and vaccine programme managers evaluate whether they are providing the vaccine safely and effectively.

A number of studies have measured coverage levels for rotavirus vaccine in developed countries, and a few have looked at compliance with the recommended schedule or factors that contribute to coverage. ${ }^{11-16}$ Previous Canadian studies have measured coverage for publicly funded rotavirus vaccination ${ }^{13} 17$ using self-report data (eg, surveys) or a sample of electronic medical records from physicians to measure vaccination. However, these sampling techniques limit the size of the cohort and may bias the results. A comprehensive population-based immunisation repository can provide a more accurate measurement of rotavirus vaccine coverage and compliance levels, and an unbiased assessment of the factors that contribute to rotavirus vaccine uptake.

Therefore, the objective of this study was to use a province-wide immunisation repository to assess rotavirus coverage and schedule compliance in Alberta during the publicly funded vaccine programme. Specifically, we:

1. Measured rotavirus vaccine coverage according to the recommended schedule, including those who received at least one dose (series initiation) and at least two doses (series completion).

2. Compared rotavirus vaccine coverage to coverage for a vaccine with the same administration schedule, namely diphtheria-tetanus-pertussis-polio-Haemophilus influenzae type $b$ vaccine (DTaP-IPV-Hib).

3. Determined non-compliance with the recommended schedule, in particular the percentage of vaccinations that occurred outside the recommended schedule.

4. Determined factors associated with rotavirus uptake in Alberta.

\section{METHODS}

\section{Setting}

This retrospective cohort study took place in Alberta, a Canadian province with over 4.1 million residents, of which $99 \%$ are registered with the province's universal, publicly funded health insurance plan. This plan offers Albertans a variety of healthcare services, including access to routine publicly funded vaccines. These vaccines are available according to a schedule set by the Ministry of Health. During the time of this analysis, RV1 was publicly funded in Alberta and recommended to be administered to children at 2 and 4 months of age. Similarly, the first two doses of the DTaP-IPV-Hib vaccine are also recommended for children at 2 and 4 months of age. The Alberta Ministry of Health policy requires the first dose of rotavirus be given between 6 weeks 0 days and 19 weeks 6 days weeks of age, and series completion (two doses) before eight calendar months ( 8 months, 0 days since date of birth) of age, with at least 4 weeks between doses. Starting 1 May 2018 the province of Alberta, along with the rest of Canada, switched to the three dose RV5; however, this change occurred after the end date for our analysis.

\section{Cohort and data sources}

We built our cohort and determined vaccination status using linked health administrative data available at the Alberta Ministry of Health. Using a registry of all births in Alberta (Vital Statistics Registry), we created a retrospective population-based cohort of Albertans born in the province between 1 June 2015 and 31 August 2016. From this registry, we also obtained information (at birth) on maternal age and marital status, number of children in the household, postal code, sex and gestational age (ie, full term or preterm). Furthermore, we estimated the location of residence (ie, urban/rural) and income quintile using postal code at time of birth and 2011 Canadian census data. At birth, Albertans are registered with the Alberta Health Care Insurance Plan, which includes a unique lifetime identifier that we used to link administrative health records for that individual, including their immunisation records. The Immunisation and Adverse Reactions to Immunisation repository records every publicly delivered vaccine dose in the province, with a few notable exceptions, including individuals who receive healthcare 
in Lloydminster (healthcare administered by neighbouring province) and vaccines given on First Nations reserves (delivered outside the provincial health system). To accurately measure rotavirus vaccine coverage, we excluded any child who died or departed the province before they turned 8 months old, those born in Lloydminster, and/or any child identified as First Nations. Historical RV5 doses (eg, out of province doses, or privately provided doses) were included in our analysis when they were documented in the provincial repository or the pharmaceutical information network database, which captures all pharmaceuticals dispensed in the province.

\section{Patient and public involvement}

Patients and the public were not directly involved at any stage in the research process. However, clinicians and policy advisors were involved as coinvestigators/authors throughout the study conception, design and interpretation of findings.

\section{Data analysis}

In the first stage of the analysis, we calculated the number and percentage of doses considered 'non-compliant' with the recommended rotavirus schedule. Non-compliant doses included (1) first dose too early (ie, before 6 weeks of age), (2) first dose too late (ie, on or after 20 weeks of age), (3) second or third dose too late (ie, after eight calendar months of age), (4) dosing interval too short (ie, $<4$ weeks between doses) and (5) too many doses (ie, received more than two doses of RV1).

For our birth cohort (ie, babies born between 1 June 2015 and 31 August 2016), we calculated the coverage for rotavirus vaccine series initiation and completion from 1 February 2016 to 31 April 2017. We defined series initiation as the percent of the cohort who received at least one dose of rotavirus vaccine between 6 weeks and 8 months of age. Series completion was defined as the percent of the cohort who received at least two doses of rotavirus vaccine between 6 weeks and 8 months of age, with at least 4 weeks between doses. Therefore, first doses given too early, and second doses given too late or without the appropriate dosing interval were considered invalid, and not counted as a dose in the coverage estimates. Furthermore, we calculated the first (at least one dose) and second dose (at least two doses) coverage for DTaP-IPV-Hib at 8 months old, allowing us to compare coverage levels of two vaccines with the same recommended age of administration, that is, 2 and 4 months. For consistency, we only included doses of DTaP-IPV-Hib administered between 6 weeks and 8 months of age, even though, unlike rotavirus vaccine, there is no contraindication to late administration.

Using two separate multivariate logistic regression models, we compared the characteristics of individuals who received rotavirus vaccination (series initiation and completion) to those who were not vaccinated for rotavirus. We calculated adjusted ORs (aORs) and 95\% CIs to test the association between vaccination status and eight different independent variables captured at birth. The independent variables (all categorical) tested were year of birth, sex, gestational age, maternal age, maternal marital status, number of children in the household, urban/ rural residency and income quintile. Urban/rural residence was based on Alberta Health Services geographic standards ${ }^{18}$ with metro centres, metro influenced areas, urban and moderate urban influenced defined as 'urban' and large rural centres, rural areas and remote defined as 'rural'. Any potential interactions were tested based on clinical plausibility and findings from previous analyses. ${ }^{19}$ We used SAS V.9.4 to conduct all analyses.

\section{RESULTS}

The final cohort included 66689 children and 112743 rotavirus vaccine doses. We excluded any child who cancelled their Alberta Health insurance before they turned 8 months $(\mathrm{n}=288)$, was born in Lloydminster $(n=44)$, or who identified as First Nations $(n=3759)$. See table 1 for the demographic characteristics of the cohort. Rotavirus vaccine coverage for $\geq 1$ dose (series initiation-86.9\%) and $\geq 2$ doses (series completion-82.5\%) was consistently lower than the $\geq 1$ dose $(94.1 \%)$ and $\geq 2$ doses (91.9\%) DTaP-IPV-Hib coverage at 8 months (figure 1). Overall, the per dose non-compliance level was very low $(\sim 0.11 \%)$. Non-compliance ranged from children receiving their first dose between 41 and 20 days too early (median: 28 days), or 1 and 87 days too late (median: 11 days); or receiving second dose vaccination up to 4 months too late (median: 2.5 months). Individuals were most likely to be non-compliant with the first dose than the second dose (table 2). However, non-compliance did affect series completion; for instance, while only a very small percentage of individuals received their first dose of rotavirus vaccine late $(0.15 \%)$, the majority $(72.5 \%)$ of these individuals did not complete their rotavirus vaccine series.

A variety of factors influenced the likelihood of a child not being vaccinated for rotavirus and were consistent for both rotavirus vaccine series initiation and completion (table 3). Unmarried mothers (aOR 1.12, 95\% CI 1.06 to $1.17)$ or those who had a greater number of children in their household at time of birth (four or more in comparison to one, aOR 5.99, 95\% CI 5.57 to 6.44 ) had greater odds of not completing the rotavirus vaccine series. Similarly, children who were born preterm, in comparison to full term, had greater odds of not completing the series (aOR $1.26,95 \%$ CI 1.15 to 1.39 ). When compared with mothers over 40 years of age, those aged $31-35$ (aOR 0.86 , $95 \% \mathrm{CI} 0.75$ to 0.98 ) and $36-40$ (aOR $0.77,95 \%$ CI 0.67 to 0.89 ) had significantly lower odds of not completing the rotavirus vaccine series, while mothers $21-25$ years of age (aOR $1.82,95 \%$ CI 1.58 to 2.10 ) and under 21 years of age (aOR $2.58,95 \%$ CI 2.18 to 3.05 ) had significantly greater odds of not having their children fully vaccinated. In comparison, sex did not influence the likelihood of rotavirus vaccine series completion. Similar associations were found between all variables and vaccine series initiation. 


\begin{tabular}{|c|c|}
\hline Variable & n (\%) \\
\hline \multicolumn{2}{|l|}{ Total number in the cohort $(n=66689)$} \\
\hline \multicolumn{2}{|l|}{ Vaccine coverage } \\
\hline First dose rotavirus vaccine & $57950(86.90)$ \\
\hline Second dose rotavirus vaccine* & $55035(82.52)$ \\
\hline First dose DTaP-IPV-Hib vaccine & $62757(94.10)$ \\
\hline Second dose DTaP-IPV-Hib vaccine & $61311(91.94)$ \\
\hline \multicolumn{2}{|l|}{ Income } \\
\hline Q1 & $12146(18.35)$ \\
\hline Q2 & $11610(17.54)$ \\
\hline Q3 & $10914(16.49)$ \\
\hline Q4 & $14726(22.25)$ \\
\hline Q5 & $16792(25.37)$ \\
\hline Missing & $501(0.01)$ \\
\hline \multicolumn{2}{|l|}{ Location of residence } \\
\hline Urban & $54344(82.11)$ \\
\hline Rural & $11844(17.89)$ \\
\hline Missing & $501(0.01)$ \\
\hline \multicolumn{2}{|l|}{ Maternal age (in years) } \\
\hline Under 21 & $2115(3.17)$ \\
\hline $21-25$ & $9526(14.28)$ \\
\hline $26-30$ & $22520(33.77)$ \\
\hline $31-35$ & $22389(33.57)$ \\
\hline $36-40$ & $8543(12.81)$ \\
\hline Over 40 & $1596(2.39)$ \\
\hline \multicolumn{2}{|l|}{ No of children in the household } \\
\hline 1 & $28309(42.45)$ \\
\hline 2 & $23564(35.33)$ \\
\hline 3 & $9538(14.30)$ \\
\hline$\geq 4$ & $5278(7.91)$ \\
\hline \multicolumn{2}{|l|}{ Gestation age at birth } \\
\hline Term & 63606 (95.38) \\
\hline Preterm & $3083(4.62)$ \\
\hline \multicolumn{2}{|l|}{ Maternal marital status } \\
\hline Married & 49548 (74.65) \\
\hline Not married & $16828(25.35)$ \\
\hline Unknown & $313(0)$ \\
\hline \multicolumn{2}{|l|}{ Sex } \\
\hline Female & $32840(49.24)$ \\
\hline Male & $33849(50.76)$ \\
\hline \multicolumn{2}{|l|}{ Year } \\
\hline 2015 & $31495(47.23)$ \\
\hline 2016 & $35194(52.77)$ \\
\hline
\end{tabular}

*A small number of doses $(n=30)$ in the dataset were recorded as RV5, which were likely historical doses from out of province or private delivery of rotavirus vaccine. Therefore, to be consistent with Alberta guidelines, ${ }^{33}$ any individual who received a dose of RV 5 needed three doses of rotavirus vaccine for complete vaccination; these individuals are included in second dose coverage.

DTaP-IPV-Hib, diphtheria-tetanus-pertussis-polio-Haemophilus influenzae type $b$.
There was an interaction effect between location of residence and income, where rural children in lower income areas had lower odds of being vaccinated for rotavirus than those in higher income areas. In comparison, in urban areas, income did not affect one's odds of being vaccinated (figure 2 ).

\section{DISCUSSION}

This was one of the first studies to use a population-based immunisation repository to evaluate rotavirus vaccine schedule compliance, as well as measuring rotavirus vaccine coverage and the factors that determine uptake. Unsurprisingly, coverage levels during the publicly funded programme were significantly higher than during the privately funded programme $(86.90 \%$ vs $2.7 \%$ initiated the series, respectively). ${ }^{11}$ Other studies looking at universal rotavirus vaccine coverage have found similar or lower coverage levels in a publicly funded programme, both in Canada (series initiation: $81 \%$, series completion: $84 \%$ ) and internationally (series completion: $73 \%-83 \%){ }^{20-22}$

As highlighted in this study, vaccine coverage at 8 months was lower for rotavirus than DTaP-IPV-Hib, 7.2\% lower for $\geq 1$ dose (series initiation) and 9.2\% lower for $\geq 2$ doses (series completion). Hull et $a t^{20}$ noted a similar phenomenon in Australia, where rotavirus coverage was consistently $7 \%$ lower than other infant vaccines. Possible explanations for this variance include the fact that rotavirus vaccine is a live vaccine, and therefore there may be fewer children eligible to receive the vaccines due to contraindications (eg, mother on biologics, immunocompromising disorders). The age restrictions for rotavirus vaccination (eg, first dose must be administered by 20 weeks) may also play a factor, along with the fact that rotavirus was very recently added to the already expansive childhood vaccination programme in Alberta. However, in the UK where there are tighter rotavirus vaccine schedule restrictions, they observe similar coverage between DTaP and rotavirus vaccination. ${ }^{16}$ Possible explanations for the difference include the fact parents may be less trusting of newly added vaccines in Canada ${ }^{23}$ and are increasingly concerned about the number of vaccines their child is receiving, and therefore may opt out of the vaccines they consider 'less important'. ${ }^{24}{ }^{25}$ Moreover, in Canada when clinicians were asked which of the newer vaccines they considered important, they ranked rotavirus as one of the lowest ${ }^{26}$ suggesting they may be less likely to recommend rotavirus vaccination for children. Future research should explore reasons for the difference in DTaP-IPV-Hib and rotavirus coverage rates, and use this evidence to improve rotavirus coverage levels.

We observed a marked difference in the levels of schedule non-compliance for rotavirus vaccination during the publicly funded programme in comparison to the privately funded programme. Specifically, during the privately funded period, MacDonald et $a l^{19}$ estimated that $7.9 \%$ of children received their vaccine too early (before 


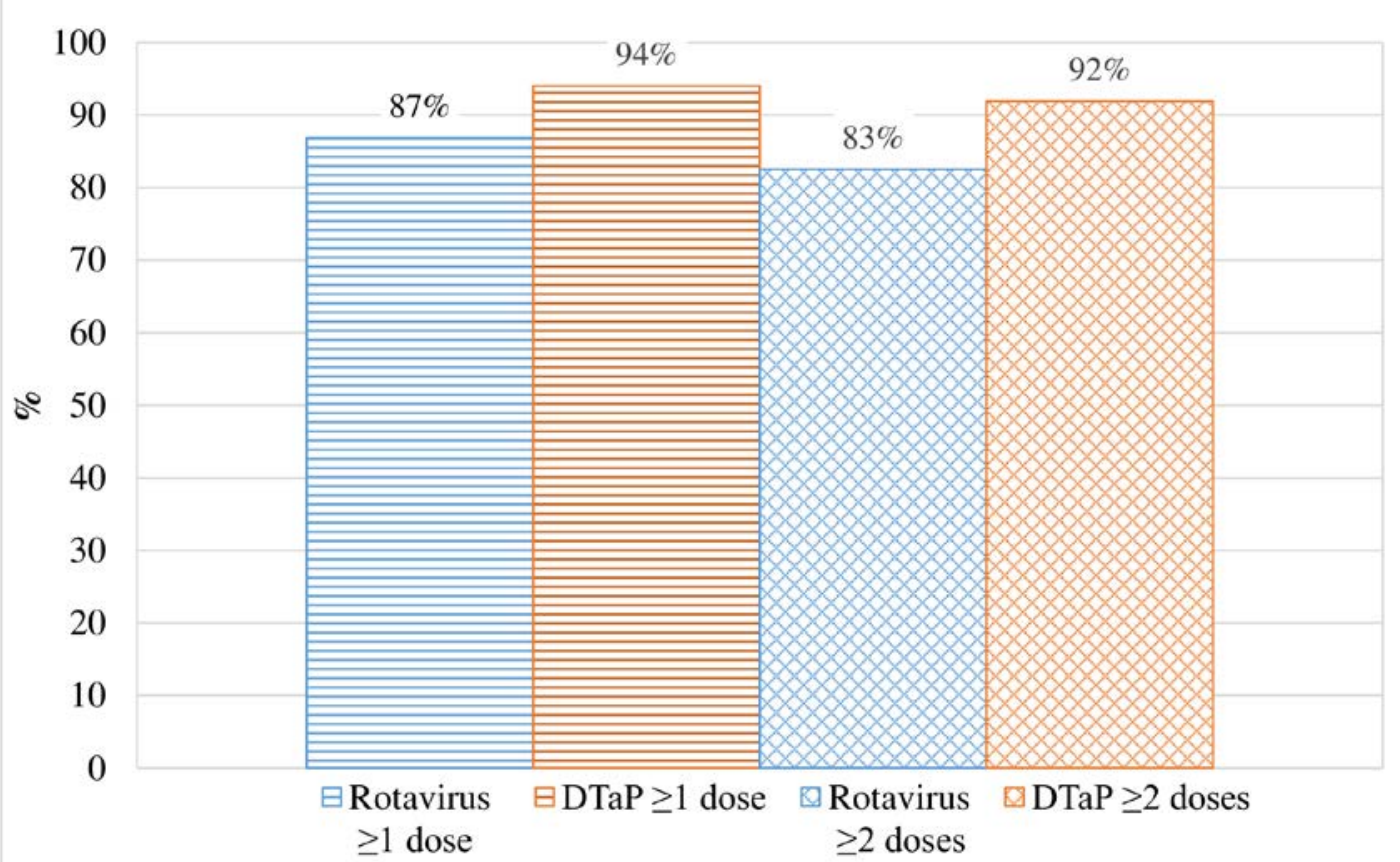

Figure 1 Alberta rotavirus and DTaP-IPV-Hib coverage rates ( $\geq 1$ dose and $\geq 2$ doses), 2016-2017. DTaP-IPV-Hib, diphtheriatetanus-pertussis-polio-Haemophilus influenzae type $b$.

6 weeks) and $3.8 \%$ received it too late (after 32 weeks); in comparison, during the publicly funded period $<0.1 \%$ of doses were given too early or too late. One explanation for the disparate non-compliance levels may be differences in vaccine provider; specifically, changing from physicians in the privately funded period to public health nurses in the publicly funded period. Public health nurses provide the vast majority of childhood vaccines in the province, and as such, may be more aware and knowledgeable about vaccination schedules and contraindications for specific

\begin{tabular}{|c|c|c|}
\hline Variable & Definition & $\mathrm{n}(\%)$ \\
\hline \multicolumn{3}{|l|}{ First rotavirus dose non-compliant ${ }^{\star}$} \\
\hline First dose too early & First dose before 42 days of age & $3(0.01)$ \\
\hline First dose too late & First dose after 139 days of age & $88(0.15)$ \\
\hline Total & Either first dose too early or too late & $91(0.16)$ \\
\hline \multicolumn{3}{|l|}{$\begin{array}{l}\text { Second or third rotavirus dose non- } \\
\text { compliant } †\end{array}$} \\
\hline Dosing interval too short & $<28$ days between first-second or second-third dose & $8(0.01)$ \\
\hline Second/third doses $\ddagger$ too late & Second or third doses after 8 months of age & $18(0.03)$ \\
\hline $\begin{array}{l}\text { Three doses administered when } \\
\text { unnecessary }\end{array}$ & Three doses when only two doses are required & $12(0.02)$ \\
\hline Total & $\begin{array}{l}\text { Dosing interval too short, or second or third dose too late, or } \\
\text { three invalid doses }\end{array}$ & $38(0.05)$ \\
\hline Total non-compliant doses§ & Any non-compliant dose & $129(0.11)$ \\
\hline \multirow[t]{2}{*}{ Total compliant doses } & Compliant first rotavirus vaccine doses & $57500(99.84)$ \\
\hline & Compliant second and third rotavirus vaccine doses & $55114(99.93)$ \\
\hline
\end{tabular}

*Denominator equals the total number of first doses $=57591$.

†Denominator equals the total number of second and third doses $=55152$.

$\ddagger$ A small number of doses $(n=30)$ in the data set were recorded as RV5, which were likely historical doses from out of province or private delivery of rotavirus vaccine. Therefore, to be consistent with Alberta guidelines, ${ }^{33}$ any individual who received a dose of RV5 needed three doses of rotavirus vaccine for complete vaccination.

$\S$ Denominator equals the total number of first, second and third doses $=112743$. 


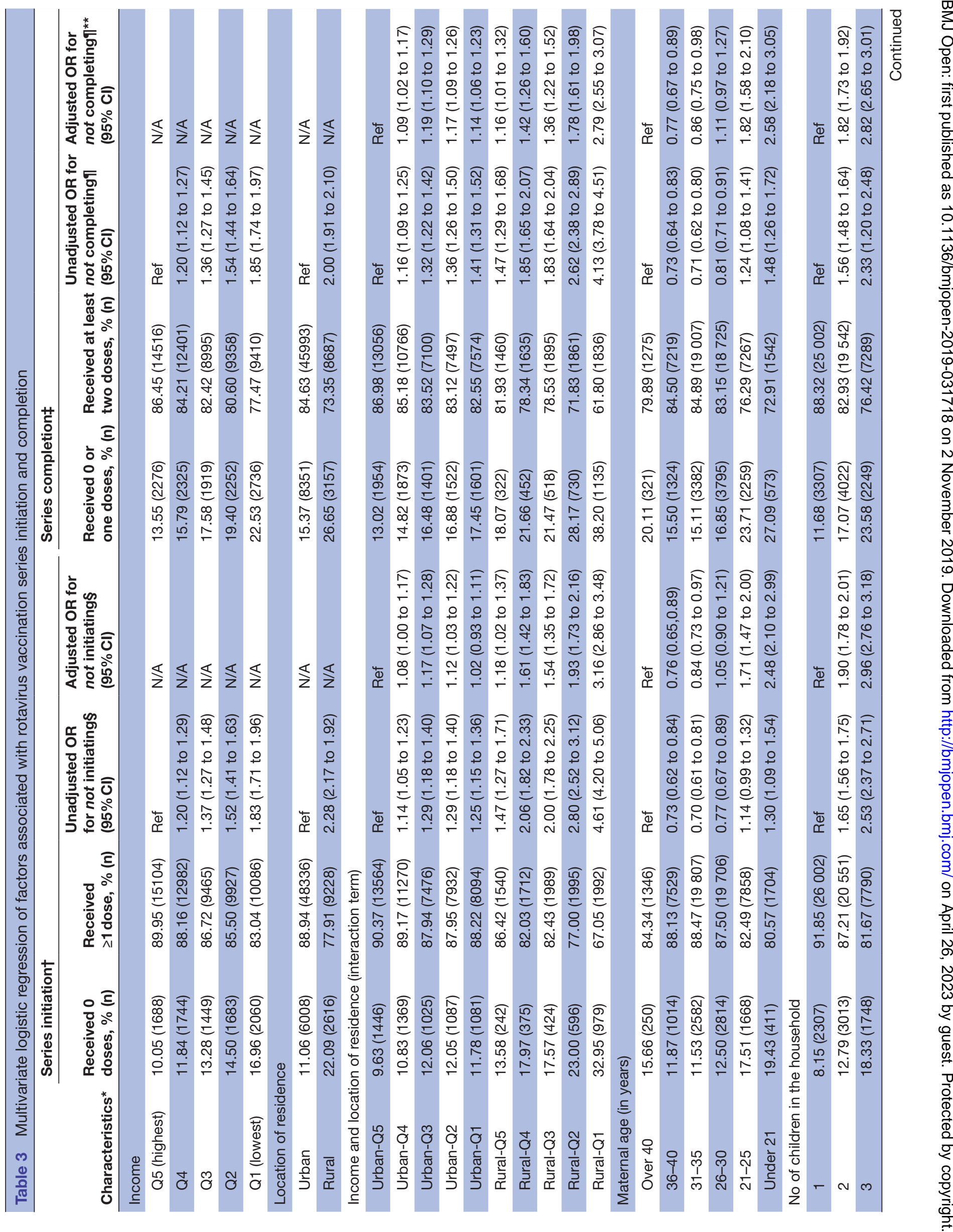




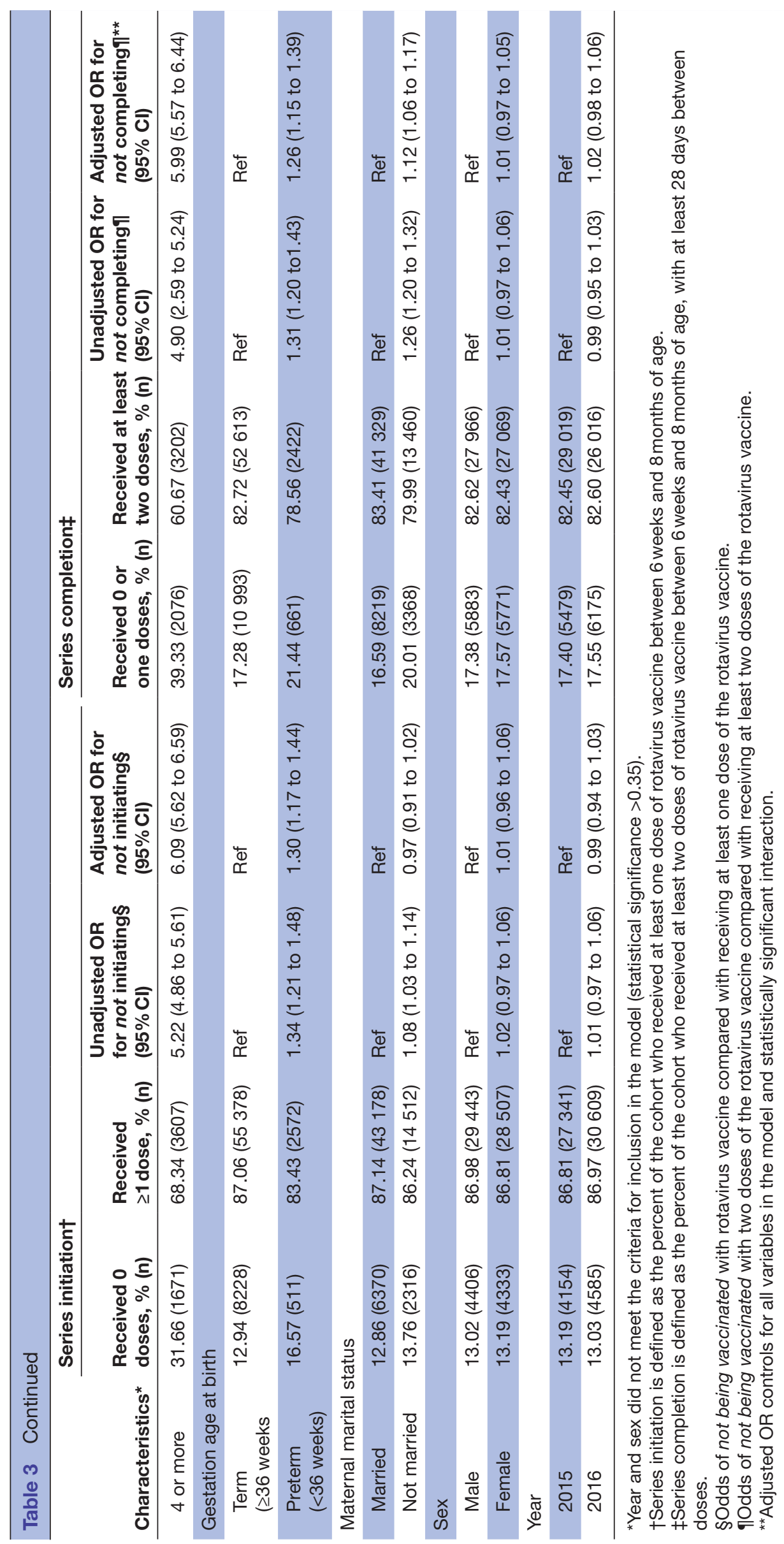




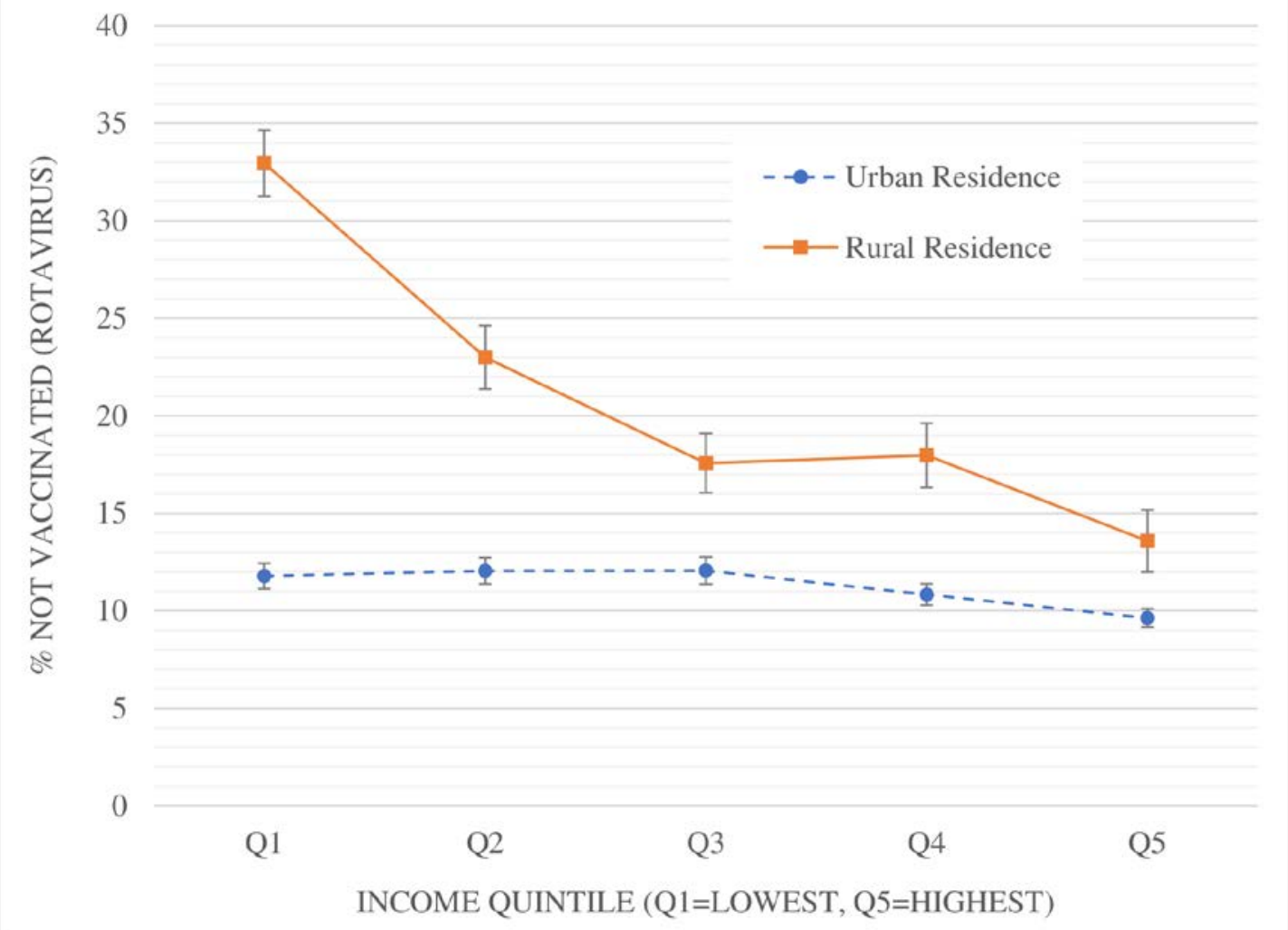

Figure 2 Rotavirus vaccine coverage (proportion of children dispensed ( $\geq 1$ dose) by income and location (urban/rural residence).

vaccines. Physician's provision of the privately funded vaccine in Alberta required parents to fill a prescription for the rotavirus vaccine and bring it to the physician for administration, and therefore, parents were partially responsible for on-time dose delivery. Moreover, in the privately funded period, most individuals purchased RV5, which required three doses and therefore increased the possibility for schedule non-compliance. ${ }^{15}$

Many of the predictive factors for rotavirus uptake remained consistent between the publicly and privately funded programmes, including number of children in the household, income and location of residence. At the same time, there were also some notable differences. For example, while preterm infants had greater odds of receiving the rotavirus vaccine during the privately funded period, ${ }^{19}$ the opposite was true during the publicly funded period. This difference may be because public health nurses do not provide RV1 to infants in the Neonatal Intensive Care Unit, which puts the onus on parents to bring their child to a public health clinic following hospital discharge (and which may be too late for receipt of the vaccine). The under-vaccination of preterm infants is an important concern because it is highly recommended they receive the rotavirus vaccine, as they are at an increased risk of severe disease. ${ }^{27}$

Similar to our findings, studies in both the $\mathrm{UK}^{28}$ and $\mathrm{USA}^{21}$ found income, social deprivation and location of residence were significant predictors for rotavirus uptake. However, we were the first to identify an interaction between location of residence and income, where income effected the odds of someone living in a rural area getting vaccinated. Canada sees similar socioeconomic disparities in vaccine uptake in other publicly available vaccines, including pertussis. ${ }^{29}$ Specifically, Gilbert $e t a t^{29}$ found lower income household, parents with lower education and children born out of Canada had higher odds of being partially immunised for pertussis. ${ }^{29}$ The low rotavirus vaccination rate in those with lower-income rural communities is particularly concerning as this population is at a higher risk of gastrointestinal illness. ${ }^{30}$ A Canadian study found that partial vaccination was more common than complete rejection, suggesting that access may be more of an issue for this population than active rejection. ${ }^{31}$ However, more research into why we see this socioeconomic and demographic discrepancy in vaccination status is needed and may identify some levers to improve coverage (eg, better public transportation links to rural health centres).

At the same time, there were some notable differences between our findings and a similar study conducted in Ontario. ${ }^{13}$ Specifically, Wilson et $a l^{13}$ did not find income, preterm birth or location of residence were significant predictors for rotavirus vaccine uptake. They also identified some additional factors that increase vaccine uptake, 
including high continuity of care, maternal influenza vaccination and maternal immigration in the last 5 years. One reason for the inconsistent findings may be sampling technique and sample size; our study included the whole Alberta population $(\mathrm{n}=66689)$, whereas Wilson et $a l^{13}$ were limited to a sample based on $3 \%$ of physicians in the province who had chosen to share their data $(n=7486)$.

We had some notable limitations in our analysis. First, we were unable to capture vaccines provided through Albertan physicians; however, individuals would have to pay for vaccines delivered from a physician and therefore we assumed a very small number of parents would choose this option. Second, we were unable to capture vaccine uptake in First Nations populations. This may be an important factor to capture in future analyses, especially as MacDonald $e t a l^{19}$ found First Nations children had significantly lower odds of being vaccinated with rotavirus during the privately funded vaccination programme. Furthermore, a study conducted in Australia found rotavirus coverage levels were $11 \%-17 \%$ lower in Indigenous infants, with more vaccination occurring offschedule in this population. ${ }^{20}$ Third, we only had approximately 1 year of data, which meant it was difficult to track changes over time. However, since we were working at the population level, we were able to establish a large sample size for the analysis.

\section{CONCLUSION}

As of 2018, many developed countries, including $40 \%$ of European countries, have no national recommendations for rotavirus vaccine, and continue to debate the benefits of adding a rotavirus vaccine to their universal vaccination programmes. ${ }^{1132}$ Our study findings provide evidence on what rotavirus vaccine coverage levels a developed country can expect to achieve with a universal vaccine programme and how those levels compare to other vaccines given on the same schedule. It is noteworthy that with tighter restrictions on age for administration of rotavirus vaccination (which is the case for RV5 in Canada, and for both RV1 and RV5 in the US and Australia), coverage levels may be lower than those observed in our study. Our study shows that a publicly funded system can achieve very high levels of schedule compliance, even with a restrictive rotavirus vaccine schedule, but highlights some populations (eg, preterm infants, low-income rural populations) that may remain under-vaccinated.

Twitter Lawrence W Svenson @Larry_Svenson and Shannon Elizabeth MacDonald @SE_MacDonald

Contributors The study was conceptualised by SM, ER and LS and designed by SM and ER. The data analysis plan was developed by SM and ER and data analysis was conducted by ER, $X G$ and $B M$. The manuscript was drafted by ER and critically revised and approved by SM, ER, XG, BM and LS.

Funding Alberta Ministry of Health, Research Agreement \#006734. Shannon MacDonald holds a career development award from the Canadian Child Health Clinician Scientist Program (CCHCSP).

Competing interests None declared.

Patient consent for publication Not required.
Ethics approval Ethics approval was obtained from the University of Alberta Human Research Ethics Board ID: Pro00078643.

Provenance and peer review Not commissioned; externally peer reviewed.

Data availability statement Data may be obtained from a third party and are not publicly available.

Open access This is an open access article distributed in accordance with the Creative Commons Attribution Non Commercial (CC BY-NC 4.0) license, which permits others to distribute, remix, adapt, build upon this work non-commercially, and license their derivative works on different terms, provided the original work is properly cited, appropriate credit is given, any changes made indicated, and the use is non-commercial. See: http://creativecommons.org/licenses/by-nc/4.0/.

ORCID iD

Lawrence W Svenson http://orcid.org/0000-0002-3391-578X

\section{REFERENCES}

1 Troeger C, Forouzanfar M, Rao PC, et al. Estimates of global, regional, and national morbidity, mortality, and aetiologies of diarrhoeal diseases: a systematic analysis for the global burden of disease study 2015. Lancet Infect Dis 2017;17:909-48.

2 World Health Organization. Rotavirus vaccines WHO position paper January 2013. Wkly Epidemiol Rec;2013:49-64.

3 Parashar UD, Cortese MM, Offit PA. Rotavirus vaccines. 7th ed. Elsevier Inc, 2018.

4 National Advisory Committee on Immunization. Literature review on rotavirus: disease and vaccine characteristics. Canada Commun Dis Rep 2010;36:1-31.

5 Morton VK, Thomas MK, McEwen SA. Estimated hospitalizations attributed to norovirus and rotavirus infection in Canada, 2006-2010. Epidemiol Infect 2015;143:3528-37.

6 Coyle D, Coyle K, Bettinger JA, et al. Cost effectiveness of infant vaccination for rotavirus in Canada. Can J Infect Dis Med Microbiol 2012;23:71-7.

7 Karafillakis E, Hassounah S, Atchison C. Effectiveness and impact of rotavirus vaccines in Europe, 2006-2014. Vaccine 2015;33:2097-107.

8 Wilson SE, Rosella LC, Wang J, et al. Population-level impact of Ontario's infant rotavirus immunization program: evidence of direct and indirect effects. PLoS One 2016;11:e0154340.

9 Hawken S, Ducharme R, Rosella LC, et al. Assessing the risk of intussusception and rotavirus vaccine safety in Canada. Hum Vaccin Immunother 2017;13:703-10.

10 Le Saux N, Canadian Pediatric Society. Position statement: recommendations for the use of rotavirus vaccines in infants, 2018. Available: https://www.cps.ca/en/documents/position/rotavirusvaccines [Accessed 6 May 2019].

11 Parez N, Giaquinto C, Du Roure C, et al. Rotavirus vaccination in Europe: drivers and barriers. Lancet Infect Dis 2014;14:416-25.

12 Braeckman T, Theeten H, Lernout T, et al. Rotavirus vaccination coverage and adherence to recommended age among infants in Flanders (Belgium) in 2012. Euro Surveil 2014;19:20806-9.

13 Wilson SE, Chung H, Schwartz KL, et al. Rotavirus vaccine coverage and factors associated with uptake using linked data: Ontario, Canada. PLoS One 2018;13:e0192809-19.

14 Panozzo CA, Becker-Dreps S, Pate V, et al. Patterns of rotavirus vaccine uptake and use in privately-insured us infants, 2006-2010. PLoS One 2013;8:e73825.

15 Calnan M, Krishnarajah G, Duh MS, et al. Rotavirus vaccination in a Medicaid infant population from four us states: compliance, vaccination completion rate, and predictors of compliance. Hum Vaccin Immunother 2016;12:1235-43.

16 Hungerford D, Vivancos R, Read JM, et al. Mitigating bias in observational vaccine effectiveness studies using simulated comparator populations: application to rotavirus vaccination in the UK. Vaccine 2018;36:6674-82.

17 Boulianne N, Audet D, Bradet R. Enquête sur La couverture vaccinale des enfants de 1 an et 2 ANS Au Québec en 2012 Institut national de santé publique Du Québec; 2013.

18 Alberta Health Services. Official standard geographic areas 2018.

19 MacDonald SE, Bell CA, Simmonds KA. Coverage and determinants of uptake for privately funded rotavirus vaccine in a Canadian birth cohort, 2008-2013. Pediatr Infect Dis J 2016;35:e177-9.

20 Hull BP, Menzies R, Macartney K, et al. Impact of the introduction of rotavirus vaccine on the timeliness of other scheduled vaccines: the Australian experience. Vaccine 2013;31:1964-9.

21 Hill HA, Elam-Evans LD, Yankey D, et al. Vaccination coverage among children aged 19-35 months - United States, 2015. MMWR Morb Mortal Wkly Rep 2016;65:1065-71. 
22 Valcarcel Salamanca B, Hagerup-Jenssen ME, Flem E. Uptake and timeliness of rotavirus vaccination in Norway: the first year postintroduction. Vaccine 2016;34:4684-9.

23 Dubé E, Bettinger JA, Halperin B, et al. Determinants of parents' decision to vaccinate their children against rotavirus: results of a longitudinal study. Health Educ Res 2012;27:1069-80.

24 Wallace AS, Mantel C, Mayers G, et al. Experiences with provider and parental attitudes and practices regarding the administration of multiple injections during infant vaccination visits: lessons for vaccine introduction. Vaccine 2014;32:5301-10.

25 Bedford H, Lansley M. More vaccines for children? Parents' views. Vaccine 2007;25:7818-23.

26 Dubé E, Gilca V, Sauvageau C, et al. Clinicians' opinions on new vaccination programs implementation. Vaccine 2012;30:4632-7.

27 Roué J-M, Nowak E, Le Gal G, et al. Impact of rotavirus vaccine on premature infants. Clin Vaccine Immunol 2014;21:1404-9.

28 Byrne L, Ward C, White JM, et al. Predictors of coverage of the National maternal pertussis and infant rotavirus vaccination programmes in England. Epidemiol Infect 2018;146:197-206.
29 Gilbert NL, Gilmour H, Wilson SE, et al. Determinants of nonvaccination and incomplete vaccination in Canadian toddlers. Hum Vaccin Immunother 2017.

30 Hungerford D, Vivancos R, Read JM, et al. Rotavirus vaccine impact and socioeconomic deprivation: an interrupted time-series analysis of gastrointestinal disease outcomes across primary and secondary care in the UK. BMC Med 2018;16:1-14.

31 Bell CA, Simmonds KA, MacDonald SE. Exploring the heterogeneity among partially vaccinated children in a population-based cohort. Vaccine 2015;33:4572-8.

32 Poelaert D, Pereira P, Gardner R, et al. A review of recommendations for rotavirus vaccination in Europe: arguments for change. Vaccine 2018;36:2243-53.

33 Province-wide immunization program standards and quality. rotavirus vaccine biological page, 2018. Available: https://www.albertahealt hservicesalbertahealthservicesalbertahealthservicesalbertahealth services.ca/assets/info/hp/cdc/if-hp-cdc-ipsm-rotavirus-vaccinebiological-page.pdf 
Correction: Measurement of coverage, compliance and determinants of uptake in a publicly funded rotavirus vaccination programme: a retrospective cohort study

Rafferty E, Guo X, McDonald B, et al. Measurement of coverage, compliance and determinants of uptake in a publicly funded rotavirus vaccination programme: a retrospective cohort study. BMJ Open 2019;9:e031718. doi: 10.1136/ bmjopen-2019-031718.

This article was previously published with errors in Table 3 .

- In table 3, the data (1.93 (1.73 to 2.16)) is for 'Rural-Q2' instead of 'Rural-Q1' under the heading 'Income and location of residence (interaction term)'.

- In table 3, the first sub-heading under 'Gestation age at birth' should read 'Term ( $\geq 36$ weeks)'. Currently, these are split into two lines.

Table 3 columns headers should read as follows:

Column 4: Unadjusted OR for not initiating\$ (95\% CI)

Column 5: Adjusted OR for not initiating $§(95 \%$ CI)

Column 8: Unadjusted OR for not completingI (95\% CI)

Column 9: Adjusted OR for not completingI, ** (95\% CI)

The footnotes to table 3 should read:

$\S$ : Odds of not being vaccinated with rotavirus vaccine compared with receiving at least one dose of the rotavirus vaccine.

If: Odds of not being vaccinated with two doses of the rotavirus vaccine compared with receiving at least two doses of the rotavirus vaccine.

Open access This is an open access article distributed in accordance with the Creative Commons Attribution Non Commercial (CC BY-NC 4.0) license, which permits others to distribute, remix, adapt, build upon this work non-commercially, and license their derivative works on different terms, provided the original work is properly cited, appropriate credit is given, any changes made indicated, and the use is non-commercial. See: http://creativecommons.org/licenses/by-nc/4.0/.

(c) Author(s) (or their employer(s)) 2019. Re-use permitted under CC BY-NC. No commercial re-use. See rights and permissions. Published by BMJ.

BMJ Open 2019;9:e031718corr1. doi:10.1136/bmjopen-2019-031718corr1

Check for updates 